\title{
Immunomodulation as a neuroprotective and therapeutic strategy for Parkinson's disease
}

\author{
Katherine E. Olson*and Howard E. Gendelman*‡ \\ *Department of Pharmacology and Experimental Neuroscience, Center for \\ Neurodegenerative Disorders, University of Nebraska Medical Center, Omaha, \\ Nebraska, 68198 \\ ${ }^{\ddagger}$ Corresponding author: Howard E. Gendelman, M.D., Department of \\ Pharmacology and Experimental Neuroscience, 985880 Nebraska Medical \\ Center, Omaha, NE 68198-5880 \\ USA; Email: hegendel@unmc.edu; phone: 01-402-559-8920; fax: 01-402-559- \\ 3744
}

Running Title: Neuroprotection for Parkinson's disease

Word count: Abstract:113 Manuscript length: 3502

\section{Number of figures: 1}

Number of references: 81

Conflict of Interest: The authors declare no conflict of interest.

Acknowledgements: This work was supported, in part, by the University of Nebraska Foundation which includes individual donations from Carol Swarts and Frances and Louie Blumkin, the Vice Chancellor's office of the University of 
Nebraska Medical Center for Core Facility Developments and National Institutes of Health grants P01 DA028555, R01 NS36126, P01 NS31492, 2R01 NS034239, P01 MH64570, P01 NS43985, P30 MH062261 and R01 AG043540. Our research is also supported by the Department of Defense grant W81XWH11-10700.

Keywords: Parkinson's disease; immunomodulation; neuroprotection; $\alpha$-syn; vaccine

Chemical compounds studied in this article:

Minocycline (CID: 54675783); 1-Methyl-4-phenyl-1,2,3,6-tetrahydropyridine (CID: 1388); 6-hydroxydopamine (CID: 4624); Resveratrol (CID: 445154); Tanshinone (CID: 114917); Silymarin (CID: 31553); Pioglitazone (CID: 4829); Rosiglitazone (CID: 77999) 


\begin{abstract}
While immune control is associated with nigrostriatal neuroprotection for Parkinson's disease, direct cause and effect relationships have not yet been realized, and modulating the immune system for therapeutic gain has been openly debated. Here, we review how innate and adaptive immunity affect disease pathobiology, and how each could be harnessed for treatment. The overarching idea is to employ immunopharmacologics as neuroprotective strategies for disease. The aim of the current work is to review disease-modifying treatments that are currently being developed as neuroprotective strategies for PD in experimental animal models and for human disease translation. The longterm goal of this research is to effectively harness the immune system to slow or prevent PD pathobiology.
\end{abstract}




\section{Introduction}

Parkinson's disease (PD) is the second most common neurodegenerative disorder, second only to Alzheimer's disease (AD). It is characterized by progressive loss of dopaminergic neurons within the nigrostriatum as well as the formation of proteinaceous inclusions of alpha-synuclein (a-syn). Secondary to the loss of dopaminergic neurons within the substanta nigra pars compacta and their connections to the striatum, there is a progressive decline in both motor and non-motor functions. The cause of the disease remains incompletely understood. However, genetics, environment, age and an interplay between the innate and adaptive immune system all contribute to disease onset and progression. To date, treatments are palliative, and there is no known cure or intervention to slow

or halt disease progression [1]. Thus, it remains essential that therapies are developed for combating PD progression rather than simply alleviating symptoms. Currently, drug therapies employ dopamine or dopamine precursor, L-DOPA, which show no effect on the neurodegenerative process. While the pathobiology of PD remains in study, neuroinflammation and immunity are thought to speed nigrostriatal degeneration $[2,3]$. The neuroinflammatory cascade associated with PD begins with aggregation of misfolded or posttranslationally modified $\alpha$-syn. Such aggregation results in neuronal cell death and the presence of chronically activated glia (microglia and astrocytes), leading to the production of tumor necrosis factor alpha (TNFa), interleukin-1 beta (IL$1 \beta$ ), IL-6, reactive oxygen species (ROS), and enzymes such as nicotinamide adenine dinucleotide phosphate (NADPH) oxidase [4]. Such changes in the 
microglial phenotype can affect the central nervous system (CNS) microenvironment by producing a pro-inflammatory milieu that speeds PD pathogenesis (Fig 1A). Compelling evidence in support of the role of neuroinflammation leading to neurodegeneration in PD is also found in human studies. Elevated plasma concentrations of proinflammatory cytokines, such as IL-6, have been correlated with a risk for PD [5], and the presence of activated microglial cells and increased levels of proinflammatory cytokines, TNF $\alpha, \mathrm{IL}-1 \beta$, IL-2, IL-6, cyclooxygenase-1 (COX-1), COX-2, and inducible nitric oxide synthase (iNOS) are found in post-mortem tissues, as well as within the cerebral spinal fluid (CSF) [6-8]. Transformation of microglia is known to occur in response to pathology. This evolves from a dynamic phenotype or classic activation to immune suppressive signatures and explains the varied functional roles of microglia as neuroprotective or neurotoxic $[9,10]$. CD8+ and CD4+ peripheral lymphocytes have also been found in post-mortem brains of PD patients, suggesting an active adaptive immune response within the substantia nigra $[11,12]$. Aside from postmortem evaluation of PD brain tissues, increased cytokine expression, changes in $\mathrm{T}$ cell ratios, and microglial activation have also been associated with and detected in multiple experimental animal models of PD, further supporting the role of immunity in PD pathogenesis [13]. Interestingly, adoptive transfer of Th1 or Th17 cells from a-syn-immunized donor mice leads to exacerbated neurodegeneration in the MPTP mouse model of PD, whereas adoptive transfer of Treg attenuates neurodegeneration by sparing dopaminergic neurons following MPTP [14]. However, CD4+CD25+ Treg isolated from mice 
immunized with $\alpha$-syn are decreased and deficient in their ability to suppress the proliferation of effector T cells (Teff) in vitro [15]. A similar observation was also observed with Treg isolated from PD patients themselves, suggesting a Treg deficit in PD [16]. All affects the tempo of disease progression. Therapeutically, these findings suggest that the development of strategies to modulate rather than contain immune responses can lead to an effective strategic outcome.

Non-steroidal anti-inflammatory drugs and minocycline Upon observational evaluation of large cohorts of individuals, those who use nonsteroidal anti-inflammatory drugs (NSAIDs), specifically ibuprofen, exhibit a lower risk of developing PD, and treatment with NSAIDs is protective in both 1-methyl4-phenyl-1,2,3,6-tetrahydropyridine- (MPTP) and 6-hydroxydopamine- (6-OHDA) induced neuronal lesions [17]. Ultimately, this suggests that there is an association with anti-inflammatory use and a decrease in the potential of developing PD. One of the first anti-inflammatory treatments to be investigated was minocycline. Minocycline possesses potent neuroprotective and antiinflammatory activity in various inflammatory rodent models of PD, and has been carried into clinical trials as well. Its mechanism of action is mediated by blocking activation of NADPH oxidase, decreasing microglial activation, and decreasing the production of iNOS, IL-1 $\beta$, and TNFa [18-22]. In both the MPTP and 6-OHDA models, minocycline reduced the numbers of reactive microglia and spared nigral dopaminergic neurons along with their projections into the striatum in a dosedependent manner. However, in the clinical setting, neuroimaging analysis of PD 
patients following minocycline treatment suggest that microglial activation is perturbed, but motor assessments failed to show any clinical benefit [23-25]. In conclusion, anti-inflammatory agents, while having been extensively studied, have failed to provide improved clinical outcomes.

\section{Natural compounds}

Many natural and/or endogenous compounds have recently been shown to be effective in rodent models of PD as well, such as resveratrol [26-28], tanshinone [29,30], silymarin [31-35], resolvins [36], diadzein [37], and apocynin derivatives $[38,39]$. The majority of these compounds act as both anti-inflammatory agents as well as anti-oxidants. Collectively, they mediate their actions by downregulating glial activation, decreasing pro-inflammatory cytokine production, suppressing M1 microglial phenotypes, and reducing NF-KB activation. Specifically, resveratrol and tanshinone treatment both show decreases in IL-1 $\beta$, IL-6, and TNFa $[27,28,30]$. Also, resveratrol causes an upregulation of suppressor of cytokine signaling (SOCS-1), and tanshinone treatment inhibited nitric oxide (NO) production, as well as NADPH and iNOS expression. In the MPTP model, silymarin treatment stabilizes mitochondrial membrane potential, decreases levels of TNFa, iNOS, and IL-1 $\beta$, and ultimately, decreases striatal levels of caspase-3 and NF-KB indicating an anti-apoptotic and anti-inflammatory function $[32,34,35]$. Taken together, these findings help to justify the potential beneficial use of natural anti-inflammatory agents for the treatment of PD. However, these agents have yet to be translated into clinical investigation for 
efficacy in PD patients.

\section{Peroxisome proliferator-activated receptor agonists}

To date, there have been many recent investigations into the role of peroxisome proliferator-activated receptor (PPAR) agonists and their neuroprotective mechanisms within the CNS. PPAR is a receptor super family of transcription factors that is activated by small molecules such as hormones and steroids, and its activation plays an important role in mediating the inflammatory response. PPAR agonists, such as pioglitazone and rosiglitazone, are shown to possess neuroprotective and anti-inflammatory activity both in vitro and in vivo by selectively targeting neurotoxic factors within reactive microglia $[40,41]$. Their use in the MPTP model of PD led to decreases in microglial activation, iNOS production, NF-KB activation, and ROS production [40,42-45]. Along with a decrease in pro-inflammatory mediators, it is associated with increases in antiinflammatory mediators such as IL-10 and transforming growth factor- $\beta$ (TGF- $\beta$ ), as well as restored levels of CD206, a marker for anti-inflammatory M2 microglial responses [40]. These findings suggest a shift from a pro-inflammatory response to an anti-inflammatory one, perhaps leading to a more protective and neurotrophic environment. To further confirm neuroprotective effects and the ability to modulate the inflammatory response, pioglitazone was tested in parkinsonian monkeys. Oral administration led to higher stereological counts of tyrosine hydroxylase-positive neurons and modulated CD68+ inflammatory cells in a dose-dependent manner [46]. To this end, these findings have prompted 
translation of PPAR agonists, specifically pioglitazone, into a multi-center, double-blind phase 2 clinical trial [47]. This trial also attempted to find peripheral biomarkers for PD that included leukocyte PGC-1a, IL-6, and urine 8hydroxydeoxyguanosine [48]. There were no changes in peripheral biomarkers observed. Additionally, the agonist failed to show benefit by reduced Unified Parkinson's Disease Rating Scale Part III (UPDRS III) scores. Explanations for why PPAR agonists failed in human PD studies likely reflect the fact that the neurotoxin-inducing neuroinflammatory models incompletely reflect human disease. In addition, multiple inflammatory response targets are likely needed, along with secondary drug-induced neuroprotective activities. The UPDRS III scores may show other limitations. Indeed, there is the potential that using motor tests as an singular end point may not preclude other disease events that could be influenced by PD-modifying treatments. The span of time for the assessment was also relatively short. This would not allow evaluation as to whether the treatment had long-term beneficial effects. Potentially, the anti-inflammatory activities of PPAR agonists may slow the rate of progression in the long term. However, the lack of observed efficacy in treating motor dysfunction should prompt researchers to continue to explore other therapeutic alternatives.

Due to the lack of clinical efficacy using anti-inflammatory agents, potential therapies aimed at directly modulating immune aberrancies are actively being studied and investigated in the clinical setting. Currently, two main therapeutic 
avenues are being explored and have shown promise in both rodent models of disease and PD itself. These two methods are based on the specific targeting of aberrantly misfolded proteins and peripheral immune modulation to elicit immune transformation into a protective phenotype.

\section{Targeting alpha-synuclein}

This therapeutic strategy is aimed at ameliorating PD-associated neuroinflammation and disease progression via targeting misfolded a-syn. In recent years, it has been shown that toxic versions of a-syn can accumulate within neurons and be secreted into the extracellular environment where the misfolded, oligomeric protein can cause a neurotoxic, inflammatory immune response. Thus, immunotherapeutic approaches are being developed to target and enhance clearing of misfolded or post-translationally modified a-syn. There is potential benefit in targeting both the $\mathrm{N}$-terminal and/or C-terminal region of the protein. A recent study using an AAV- $\alpha$-syn rat model of PD indicates that antihuman $\alpha$-syn $\mathrm{N}$-terminal peptide antibodies can protect against dopaminergic neuron loss and decrease microglial activation to some extent. Treatment with the vaccine also lead to altered IgG production, enhanced MHCII expression, and increased CD4+ T cell infiltration into the CNS [49]. Recently, it has also been shown that use of monoclonal antibodies against the C-terminal region of $\alpha$ syn or misfolded a-syn reduces levels of protein propagation and improves PDlike pathologies, ameliorates dopaminergic neuronal cell loss, and attenuates motor deficits in mouse models of the disease [50-54]. Based on these findings, 
in 2013, Roche and Prothena further developed and commercialized PRX002 to specifically target $\alpha$-syn. A phase I clinical trial was initiated with the finding that the vaccine was safe and well-tolerated, prompting a second phase I trial to assess dose response, tolerability, pharmacokinetics, exploratory biomarkers, and immunogenicity (NCT02095171 and NCT02157714). With a similar directive in mind, a current study using a vaccine against $\alpha$-syn demonstrated that vaccination prevented Parkinson-like inclusions, as well as increased Treg recruitment, increased glial cell-derived neurotrophic factor (GDNF) production, and increased antibody formation [55]. Excitingly, another vaccine that has been developed to produce antibodies that are specific for $\alpha$-syn while sparing neuroprotective beta-synuclein, AFFITOPE PD01A, has entered clinical trials [56,57]. PD01 targets the phosphorylated form of $\alpha$-syn that is responsible for induction of a neurotoxic proinflammatory cascade through microglial activation. The first phase is completed and has indicated that the vaccine is safe and tolerable in two doses, 15 and 75 ug (NCT01568099). Half of the patients developed $\alpha$-syn antibodies suggesting that the vaccine is effective as well. However, further investigations are underway to test PDO1A's clinical benefit in PD, as well as other parkinsonian-associated diseases [58] (NCT02216188 and NCT02270489). Collectively, these studies show that a-syn-targeted immunotherapies may have the potential to attenuate neuroinflammatoryassociated neurotoxicities and as such, delay dopaminergic neurodegeneration associated with PD. Alternatively, targeting a-syn may have some potential pitfalls. Vaccination and antibody-mediated therapies are associated with 
antigen-specific $T$ cell responses that may contribute to an inflammatory state operative during PD. This concern was realized in studies of immunotherapies for Alzheimer's disease. Here, extensive $T$ cell reactivity and an autoimmune meningoencephalitis followed immune-stimulating strategies for amyloid beta (Aß) clearance [59]. This, coupled with the presence of intracellular misfolded proteins, makes it imperative to generate vaccination designs that are longlasting and highly specific for $\alpha$-syn. The robustness of the immune response generated needs to be evaluated together with any potential cross-reactivity of common nonpathogenic forms of $\alpha$-syn or members of its protein families.

\section{Treg-mediated immune suppression}

Lastly, since there has been little success in anti-inflammatory therapy translation into the clinic, therapeutic strategies aimed at modulating the aberrant immune response during disease progression may be more appropriate. The majority of immunotherapies are designed to protect the host from something foreign; however, in neurodegenerative diseases, the target is a self-protein, making the type and duration of the peripheral immune response elicited extremely important. In the acute MPTP model of PD, nitrated $\alpha$-syn enhances the neurotoxic element of microglia activation, ultimately leading to neuronal cell loss [60]. In PD, our data supports that with inflammation and chronic immune stimulation in the brain, there is an increase in effector and memory $\mathrm{T}$ cell populations that can secrete proinflammatory and neurotoxic cytokines $[2,3]$. This can drive microglial reactivity, producing even greater levels of proinflammatory 
mediators (Fig. 1A). Thus, inhibiting Teff subsets, along with microglial activation may be of clinical benefit [61]. One such strategy utilizes the immune suppressing capabilities of Tregs to target neuroinflammation (Fig. 1B). In the MPTP mouse model, our lab has shown that Tregs can attenuate microgliosis, protect against dopaminergic neuronal death, and spare striatal termini $[14,15,62]$. Tregs can elicit a down-regulation of pro-inflammatory mediators such as iNOS, TNFa, IL-1 $\beta$, interferon gamma (IFN- $\gamma$ ), as well as decrease levels of ROS production and NF-KB activation $[63,64]$. Through these mechanisms, they may also have the ability to mediate their protective actions by switching microglial responses from a neurotoxic M1 response to a neurotrophic M2 response [15]. However, it should be noted that even resting microglia can exist in a spectrum of phenotypic states rather than solely existing in an M1 and M2 condition that are exclusively pro- or anti-inflammatory [65]. Rather, microglia may simply be shifted into a more or less anti-inflammatory state. Nonetheless, these findings indicate a phenotypic transition during Teff or Treg interactions with microglia or peripheral monocyte-macrophages. Furthermore, Tregs can act directly on activated Teff populations and antigen presenting cells by cytolysis, metabolic disruption, inhibiting maturation, and/or secreting suppressive cytokines. However, in the overall PD population, there is a Treg deficit and an increase in effector $\mathrm{T}$ cell populations that are associated with increased movement disorder [16], indicating a need for a therapy that may induce or enhance Treg functions. One appropriate therapeutic avenue would be to enhance Treg numbers or function through the use of potent immune modulating 
agents such as vasoactive intestinal peptide (VIP), pituitary adenylate cyclaseactivating polypeptide (PACAP), and granulocyte-macrophage colony stimulating factor (GM-CSF), or through the use of vaccine strategies that induce Treg populations. Notably, the use of PACAP, VIP, GM-CSF, and a Bacillus CalmetteGuerin vaccination have shown promising results in PD models. Such therapeutic interventions have shown effectiveness in the treatments of other chronic inflammatory conditions and as such, support their ability to restore immune homeostasis and repair tissue injuries [14,62,66-72]. For instance, adoptive transfer of VIP- or GM-CSF-induced Tregs following MPTP intoxication leads to enhanced neuronal survival of nearly $100 \%$, and a decrease in microglia activation [14,62]. Thus, our lab is developing an immunomodulatory strategy that controls neurotoxic inflammation associated with PD through the use of antiinflammatory and Treg-mediated immune suppression. Based on pre-clinical evaluation of these endogenous agents, our lab is currently evaluating the safety and efficacy of GM-CSF as a therapy for PD in a phase I clinical trial in order to help restore Treg dysfunction associated with PD (NCT01882010). Evaluation of potential of biomarkers, modulations in neuroimaging, and/or changes in motor assessments with treatment is also being carried out. The potential use of this therapeutic strategy, along with clearance of aberrant protein, could possibly lead to induction of Tregs that will induce a phenotypic shift in effector subsets and microglia, ultimately ameliorating disease. Although, there remains the possibility that the neuroinflammatory response that is occurring with progressed PD will not be ameliorated simply through the induction of Tregs. Tregs may not have the 
ability to suppress the detrimental immune response to a point in which protection is observed. However, pre-clinical studies have already shown potential promise and the ability of effector to regulatory cell transformation.

\section{Future directions}

In summary, progressive neurodegenerative disorders present a challenge for developing treatments because onset of the disease may be unknown, and the exact mechanism of disease initiation is poorly understood. For instance, in PD, $50-70 \%$ of the neurons within the nigrostriatal lesion may be lost before motor symptoms arise, making disease initiation difficult to combat [1]. However, neuroinflammation associated with activated microglial responses is widely considered to participate in the pathogenesis of $\mathrm{PD}$, making reactive microglia a viable therapeutic target. The pro-inflammatory cytokines and chemokines and reactive oxygen and nitrogen species secreted by reactive microglia trigger a neurotoxic cascade that has detrimental effects within the CNS by exacerbating neuronal lesions (Fig. 1A). Thus, therapies aimed at targeting neuroinflammation either directly or indirectly should be explored. Many anti-inflammatory agents and immune modulating compounds discussed in this review share this commonality and have shown promising results in experimental models of the disease. However, even with their success in animal models, there have not been clinical advances using these agents. This may be due to the fact that there is no animal model of PD that encompasses all aspects of PD. Although some therapies have shown the potential to be translated into a clinical setting, they 
may not be as successful in treating patients with progressing PD. In acute, inflammatory models of PD, targeting inflammation specifically using antiinflammatory agents has been shown to be neuroprotective, but with the multifactorial and slowly progressing nature of PD in humans, simply targeting one aspect may not be effective. Perhaps a combinatorial approach may be better suited for PD therapy.

On the other hand, microglia can also have a neuroprotective effect, and they are not static. Therefore, shutting down the microglial response altogether may be detrimental as well. Microglia exist in a continuum of reactivity, and their response can be shifted based on the surrounding cytokine environment as well as interaction with the peripheral immune system. Thus, therapies aimed at shifting the microglial response from a pro-inflammatory $\mathrm{M} 1$ to an antiinflammatory M2 response without shutting down the response altogether would be better for the long-term treatment of PD. One potential way of doing this may be carried out by modulating the innate and adaptive immune system, as well as shifting peripheral $\mathrm{T}$ cell populations from pro-inflammatory, activated Teffs to anti-inflammatory phenotypes by utilizing agents that enhance Treg-mediated suppression of the immune response (Fig. 1B). However, contrary evidence suggests that Treg are detrimental to cell survival and can enhance neurodegeneration. Notably, this was shown during the acute phase of experimental stroke when Treg expansion exacerbated brain injuries [73]. It need be noted that the acute phase is a relatively short span of time and is during a 
period consisting of an intense initial inflammatory response. Throughout this period, such an innate response in affected brain tissue is required to enhance cellular debris phagocytosis that can explain the coincident increase in inflammation associated with Treg infiltration. In contrast, another study demonstrated that IL-10-producing Tregs elicited a reduction in infarct size and attenuated the inflammatory response associated with ischemic stroke [74]. Yet another group indicates that brain derived neurotrophic factor (BDNF)-positive Treg cells are increased in stroke patients, and this increase is associated with improved clinical outcomes [75]. Further support of Treg as neuroprotective cells is observed in tests done in an experimental model of amyotrophic lateral sclerosis (ALS) and in human disease. Tregs were shown to mediate disease progression, and actively contribute to a neuroprotective cascade through their interactions with microglial populations [76-78]. Recently, a study was carried out using bee venom phospholipase $\mathrm{A} 2$ to protect dopaminergic neurons through the modulation of the inflammatory response associated with MPTP intoxication [79]. The modulation was made following Treg treatment transfer. Excitingly, when endogenous Tregs were blocked via anti-CD25 antibody, the protective phenotype was no longer observed, indicating their specific involvement. Collectively, these findings further support a direct neuroprotective role of Tregs in neurodegenerative disorders.

In regards to PD, $\alpha$-syn targeted immunotherapies and Treg-mediated therapies have undoubtedly shown considerable promise in improving disease outcomes in models of nigrostriatal degeneration. Tregs' abilities to modulate the peripheral 
immune response, as well as target innate microglial responses, represents a therapeutic avenue that may have more promising clinical benefit than other antiinflammatory agents. These other agents failed to show efficacy in the first phases of clinical trial. The mechanism by which the immune system can be used to target and control neuroinflammation is now being realized. The therapeutic potential of immunomodulating therapies is an exciting advance and raises a real possibility for successfully combating disease and changing the course of PD neurodegeneration. 
Figure 1. Immune modulation in Parkinson's disease. A) The pathogenesis of PD is affected by modified a-syn released extracellularly when dopaminergic neurons become damaged or die. The modified aggregated protein elicits microglial immune responses leading to a neurotoxic cascade within the substantia nigra pars compacta. Reactive microglia secrete neurotoxic factors that include proinflammatory mediators further accelerating neuronal cell death. Drainage of modified $\alpha$-syn across the blood-brain barrier and into secondary lymphoid tissues causes an aberrant activation of the adaptive immune system. In response to $\alpha$-syn, infiltrating effector T cells exacerbate neurodegeneration by contributing to the inflammatory neurotoxic cascade through the secretion of factors that include TNF- $\alpha$ and IFN- $\gamma$. B) The use of agents that induce Treg promote neurotrophic support and ultimately, neuronal cell survival by suppressing effector $\mathrm{T}$ cell activation, proliferation, secretion of neurotoxic mediators, and infiltration into the brain. These events modulate microglial reactivity by secreting immune suppressive factors such as IL-10, IL-4, and FASL (Fas ligand). The immune events temper neurotoxic innate and adaptive immune responses associated with disease progression, ultimately leading to a neuroprotective phenotype within the affected nervous system. 


\section{References}

1. Olanow CW, Stern MB, Sethi K: The scientific and clinical basis for the treatment of Parkinson disease (2009). Neurology 2009, 72:S1-136.

2. Mosley RL, Hutter-Saunders JA, Stone DK, Gendelman HE: Inflammation and adaptive immunity in Parkinson's disease. Cold Spring Harb Perspect Med 2012, 2:a009381.

3. Anderson KM, Olson KE, Estes KA, Flanagan K, Gendelman HE, Mosley RL: Dual destructive and protective roles of adaptive immunity in neurodegenerative disorders. Transl Neurodegener 2014, 3:25.

4. Liu B, Hong JS: Role of microglia in inflammation-mediated neurodegenerative diseases: mechanisms and strategies for therapeutic intervention. J Pharmacol Exp Ther 2003, 304:1-7.

5. Chen H, O'Reilly EJ, Schwarzschild MA, Ascherio A: Peripheral inflammatory biomarkers and risk of Parkinson's disease. $A m \mathrm{~J}$ Epidemiol 2008, 167:90-95.

6. Mogi $M$, Harada $M$, Narabayashi $H$, Inagaki $H$, Minami $M$, Nagatsu $T$ : Interleukin (IL)-1 beta, IL-2, IL-4, IL-6 and transforming growth factoralpha levels are elevated in ventricular cerebrospinal fluid in juvenile parkinsonism and Parkinson's disease. Neurosci Lett 1996, 211:13-16.

7. Mogi M, Harada M, Riederer P, Narabayashi H, Fujita K, Nagatsu T: Tumor necrosis factor-alpha (TNF-alpha) increases both in the brain and in the cerebrospinal fluid from parkinsonian patients. Neurosci Lett 1994, 165:208-210. 
8. Hunot S, Brugg B, Ricard D, Michel PP, Muriel MP, Ruberg M, Faucheux BA, Agid Y, Hirsch EC: Nuclear translocation of NF-kappaB is increased in dopaminergic neurons of patients with parkinson disease. Proc Natl Acad Sci U S A 1997, 94:7531-7536.

9. Hanisch UK, Kettenmann $\mathrm{H}$ : Microglia: active sensor and versatile effector cells in the normal and pathologic brain. Nat Neurosci 2007, 10:13871394.

10. Colton CA: Heterogeneity of microglial activation in the innate immune response in the brain. $J$ Neuroimmune Pharmacol 2009, 4:399-418.

11. Brochard V, Combadiere B, Prigent A, Laouar Y, Perrin A, Beray-Berthat V, Bonduelle O, Alvarez-Fischer D, Callebert J, Launay JM, et al.: Infiltration of CD4+ lymphocytes into the brain contributes to neurodegeneration in a mouse model of Parkinson disease. J Clin Invest 2009, 119:182-192.

12. McGeer PL, Itagaki S, Boyes BE, McGeer EG: Reactive microglia are positive for HLA-DR in the substantia nigra of Parkinson's and Alzheimer's disease brains. Neurology 1988, 38:1285-1291.

13. Kurkowska-Jastrzebska I, Wronska A, Kohutnicka M, Czlonkowski A, Czlonkowska A: MHC class II positive microglia and lymphocytic infiltration are present in the substantia nigra and striatum in mouse model of Parkinson's disease. Acta Neurobiol Exp (Wars) 1999, 59:1-8.

14. Reynolds AD, Stone DK, Hutter JA, Benner EJ, Mosley RL, Gendelman HE: Regulatory $\mathrm{T}$ cells attenuate Th17 cell-mediated nigrostriatal 
dopaminergic neurodegeneration in a model of Parkinson's disease. J Immunol 2010, 184:2261-2271.

15. Reynolds AD, Banerjee R, Liu J, Gendelman HE, Mosley RL: Neuroprotective activities of CD4+CD25+ regulatory $\mathrm{T}$ cells in an animal model of Parkinson's disease. J Leukoc Biol 2007, 82:10831094.

16. Saunders JA, Estes KA, Kosloski LM, Allen HE, Dempsey KM, TorresRussotto DR, Meza JL, Santamaria PM, Bertoni JM, Murman DL, et al.: CD4+ regulatory and effector/memory $\mathrm{T}$ cell subsets profile motor dysfunction in Parkinson's disease. J Neuroimmune Pharmacol 2012, 7:927-938.

17. Rees K, Stowe R, Patel S, Ives N, Breen K, Clarke CE, Ben-Shlomo Y: Nonsteroidal anti-inflammatory drugs as disease-modifying agents for Parkinson's disease: evidence from observational studies. Cochrane Database Syst Rev 2011:CD008454.

18. Du Y, Ma Z, Lin S, Dodel RC, Gao F, Bales KR, Triarhou LC, Chernet E, Perry KW, Nelson DL, et al.: Minocycline prevents nigrostriatal dopaminergic neurodegeneration in the MPTP model of Parkinson's disease. Proc Natl Acad Sci U S A 2001, 98:14669-14674.

19. He Y, Appel S, Le W: Minocycline inhibits microglial activation and protects nigral cells after 6-hydroxydopamine injection into mouse striatum. Brain Res 2001, 909:187-193. 
20. Kim HS, Suh YH: Minocycline and neurodegenerative diseases. Behav Brain Res 2009, 196:168-179.

21. Lu L, Li F, Wang X: Novel anti-inflammatory and neuroprotective agents for Parkinson's disease. CNS Neurol Disord Drug Targets 2010, 9:232240.

22. Tikka T, Fiebich BL, Goldsteins G, Keinanen R, Koistinaho J: Minocycline, a tetracycline derivative, is neuroprotective against excitotoxicity by inhibiting activation and proliferation of microglia. J Neurosci 2001, 21:2580-2588.

23. A randomized, double-blind, futility clinical trial of creatine and minocycline in early Parkinson disease. Neurology 2006, 66:664-671.

24. A pilot clinical trial of creatine and minocycline in early Parkinson disease: 18-month results. Clin Neuropharmacol 2008, 31:141-150.

25. Dodel R, Spottke A, Gerhard A, Reuss A, Reinecker S, Schimke N, Trenkwalder C, Sixel-Doring F, Herting B, Kamm C, et al.: Minocycline 1year therapy in multiple-system-atrophy: effect on clinical symptoms and [(11)C] (R)-PK11195 PET (MEMSA-trial). Mov Disord 2010, 25:97107.

26. Lin TK, Chen SD, Chuang YC, Lin HY, Huang CR, Chuang JH, Wang PW, Huang ST, Tiao MM, Chen JB, et al.: Resveratrol partially prevents rotenone-induced neurotoxicity in dopaminergic SH-SY5Y cells through induction of heme oxygenase-1 dependent autophagy. Int $J$ Mol Sci 2014, 15:1625-1646. 
27. Lofrumento DD, Nicolardi G, Cianciulli A, De Nuccio F, La Pesa V, Carofiglio V, Dragone T, Calvello R, Panaro MA: Neuroprotective effects of resveratrol in an MPTP mouse model of Parkinson's-like disease: possible role of SOCS-1 in reducing pro-inflammatory responses. Innate Immun 2014, 20:249-260.

28. Zhang F, Shi JS, Zhou H, Wilson B, Hong JS, Gao HM: Resveratrol protects dopamine neurons against lipopolysaccharide-induced neurotoxicity through its anti-inflammatory actions. Mol Pharmacol 2010, 78:466-477.

29. Ren B, Zhang YX, Zhou HX, Sun FW, Zhang ZF, Wei Z, Zhang CY, Si DW: Tanshinone IIA prevents the loss of nigrostriatal dopaminergic neurons by inhibiting NADPH oxidase and iNOS in the MPTP model of Parkinson's disease. J Neurol Sci 2015, 348:142-152.

30. Wang S, Jing H, Yang H, Liu Z, Guo H, Chai L, Hu L: Tanshinone I selectively suppresses pro-inflammatory genes expression in activated microglia and prevents nigrostriatal dopaminergic neurodegeneration in a mouse model of Parkinson's disease. $J$ Ethnopharmacol 2015, 164:247-255.

31. Borah A, Paul R, Choudhury S, Choudhury A, Bhuyan B, Das Talukdar A, Dutta Choudhury M, Mohanakumar KP: Neuroprotective potential of silymarin against CNS disorders: insight into the pathways and molecular mechanisms of action. CNS Neurosci Ther 2013, 19:847853. 
32. Geed M, Garabadu D, Ahmad A, Krishnamurthy S: Silibinin pretreatment attenuates biochemical and behavioral changes induced by intrastriatal MPP+ injection in rats. Pharmacol Biochem Behav 2014, 117:92-103.

33. Haddadi R, Nayebi AM, Farajniya S, Brooshghalan SE, Sharifi H: Silymarin improved 6-OHDA-induced motor impairment in hemi-parkisonian rats: behavioral and molecular study. Daru 2014, 22:38.

34. Jung UJ, Jeon MT, Choi MS, Kim SR: Silibinin attenuates MPP(+)-induced neurotoxicity in the substantia nigra in vivo. J Med Food 2014, 17:599605.

35. Lee $Y$, Park HR, Chun HJ, Lee J: Silibinin prevents dopaminergic neuronal loss in a mouse model of Parkinson's disease via mitochondrial stabilization. J Neurosci Res 2015, 93:755-765.

36. Tian $\mathrm{Y}$, Zhang $\mathrm{Y}$, Zhang R, Qiao S, Fan J: Resolvin D2 recovers neural injury by suppressing inflammatory mediators expression in lipopolysaccharide-induced Parkinson's disease rat model. Biochem Biophys Res Commun 2015, 460:799-805.

37. Chinta SJ, Ganesan A, Reis-Rodrigues P, Lithgow GJ, Andersen JK: Antiinflammatory role of the isoflavone diadzein in lipopolysaccharidestimulated microglia: implications for Parkinson's disease. Neurotox Res 2013, 23:145-153.

38. Ghosh A, Kanthasamy A, Joseph J, Anantharam V, Srivastava P, Dranka BP, Kalyanaraman B, Kanthasamy AG: Anti-inflammatory and 
neuroprotective effects of an orally active apocynin derivative in preclinical models of Parkinson's disease. J Neuroinflammation 2012, 9:241.

39. t Hart BA, Copray S, Philippens I: Apocynin, a low molecular oral treatment for neurodegenerative disease. Biomed Res Int 2014, 2014:298020.

40. Pisanu A, Lecca D, Mulas G, Wardas J, Simbula G, Spiga S, Carta AR: Dynamic changes in pro- and anti-inflammatory cytokines in microglia after PPAR-gamma agonist neuroprotective treatment in the MPTPp mouse model of progressive Parkinson's disease. Neurobiol Dis 2014, 71:280-291.

41. Carta AR, Pisanu A: Modulating microglia activity with PPAR-gamma agonists: a promising therapy for Parkinson's disease? Neurotox Res 2013, 23:112-123.

42. Garrido-Gil P, Joglar B, Rodriguez-Perez AI, Guerra MJ, Labandeira-Garcia $\mathrm{JL}$ : Involvement of PPAR-gamma in the neuroprotective and antiinflammatory effects of angiotensin type 1 receptor inhibition: effects of the receptor antagonist telmisartan and receptor deletion in a mouse MPTP model of Parkinson's disease. J Neuroinflammation 2012, 9:38.

43. Barbiero JK, Santiago R, Tonin FS, Boschen S, da Silva LM, Werner MF, da Cunha C, Lima MM, Vital MA: PPAR-alpha agonist fenofibrate protects 
against the damaging effects of MPTP in a rat model of Parkinson's disease. Prog Neuropsychopharmacol Biol Psychiatry 2014, 53:35-44.

44. Barbiero JK, Santiago RM, Persike DS, da Silva Fernandes MJ, Tonin FS, da Cunha C, Lucio Boschen S, Lima MM, Vital MA: Neuroprotective effects of peroxisome proliferator-activated receptor alpha and gamma agonists in model of parkinsonism induced by intranigral 1-methyl-4phenyl-1,2,3,6-tetrahyropyridine. Behav Brain Res 2014, 274:390-399.

45. Lecca D, Nevin DK, Mulas G, Casu MA, Diana A, Rossi D, Sacchetti G, Carta AR: Neuroprotective and anti-inflammatory properties of a novel nonthiazolidinedione PPARgamma agonist in vitro and in MPTP-treated mice. Neuroscience 2015.

46. Swanson CR, Joers V, Bondarenko V, Brunner K, Simmons HA, Ziegler TE, Kemnitz JW, Johnson JA, Emborg ME: The PPAR-gamma agonist pioglitazone modulates inflammation and induces neuroprotection in parkinsonian monkeys. J Neuroinflammation 2011, 8:91.

47. Pioglitazone in early Parkinson's disease: a phase 2, multicentre, double-blind, randomised trial. Lancet Neurol 2015.

48. Simon DK, Simuni T, Elm J, Clark-Matott J, Graebner AK, Baker L, Dunlop SR, Emborg M, Kamp C, Morgan JC, et al.: Peripheral Biomarkers of Parkinson's Disease Progression and Pioglitazone Effects. $J$ Parkinsons Dis 2015.

49. Shahaduzzaman M, Nash K, Hudson C, Sharif M, Grimmig B, Lin X, Bai G, Liu H, Ugen KE, Cao C, et al.: Anti-human alpha-synuclein N-terminal 
peptide antibody protects against dopaminergic cell death and ameliorates behavioral deficits in an AAV-alpha-synuclein rat model of Parkinson's disease. PLoS One 2015, 10:e0116841.

${ }^{*}$ Recent study indicating the ability of anti-human $\alpha$-syn N-terminal peptide antibodies to protect against dopaminergic neuron loss and microglial activation. Treatment also had positive effects on the peripheral immune system leading to antibody formation and changes in $\mathrm{MHCll}$ expression.

50. Games D, Valera E, Spencer B, Rockenstein E, Mante M, Adame A, Patrick C, Ubhi K, Nuber S, Sacayon P, et al.: Reducing C-terminal-truncated alpha-synuclein by immunotherapy attenuates neurodegeneration and propagation in Parkinson's disease-like models. J Neurosci 2014, 34:9441-9454.

** Study suggesting that reducing the accumulation of C-terminaltruncated alpha-synuclein in Parkinson's disease-like models through vaccine immunotherapy leads to attenuation of disease pathology, reduction in accumulation of aberrant alpha-synuclein, sparing of striatal termini, and improved motor and cognitive function.

51. Lindstrom V, Fagerqvist T, Nordstrom E, Eriksson F, Lord A, Tucker S, Andersson J, Johannesson M, Schell H, Kahle PJ, et al.: Immunotherapy targeting alpha-synuclein protofibrils reduced pathology in (Thy-1)h[A30P] alpha-synuclein mice. Neurobiol Dis 2014, 69:134-143. 
52. Fagerqvist T, Lindstrom V, Nordstrom E, Lord A, Tucker SM, Su X, Sahlin C, Kasrayan A, Andersson J, Welander $\mathrm{H}$, et al.: Monoclonal antibodies selective for alpha-synuclein oligomers/protofibrils recognize brain pathology in Lewy body disorders and alpha-synuclein transgenic mice with the disease-causing A30P mutation. J Neurochem 2013, 126:131-144.

53. Tran HT, Chung $\mathrm{CH}$, Iba M, Zhang B, Trojanowski JQ, Luk KC, Lee VM: Alpha-synuclein immunotherapy blocks uptake and templated propagation of misfolded alpha-synuclein and neurodegeneration. Cell Rep 2014, 7:2054-2065.

54. Vaikath NN, Majbour NK, Paleologou KE, Ardah MT, van Dam E, van de Berg WD, Forrest SL, Parkkinen L, Gai WP, Hattori N, et al.: Generation and characterization of novel conformation-specific monoclonal antibodies for alpha-synuclein pathology. Neurobiol Dis 2015, 79:8199.

55. Sanchez-Guajardo V, Annibali A, Jensen PH, Romero-Ramos M: alphaSynuclein vaccination prevents the accumulation of parkinson disease-like pathologic inclusions in striatum in association with regulatory $\mathbf{T}$ cell recruitment in a rat model. $J$ Neuropathol Exp Neurol 2013, 72:624-645.

* Recent study demonstrating the potential use of a protective vaccine strategy that elicits its actions through induction and recruitment of 
regulatory $T$ cell populations to the neurodegenerative lesion. Results also indicate increases in neurotrophic factors and antibody production.

56. Mandler M, Valera E, Rockenstein E, Weninger H, Patrick C, Adame A, Santic R, Meindl S, Vigl B, Smrzka O, et al.: Next-generation active immunization approach for synucleinopathies: implications for Parkinson's disease clinical trials. Acta Neuropathol 2014, 127:861879.

57. Schneeberger A, Mandler M, Mattner F, Schmidt W: Vaccination for Parkinson's disease. Parkinsonism Relat Disord 2012, 18 Suppl 1:S1113.

58. Mandler M, Valera E, Rockenstein E, Mante M, Weninger H, Patrick C, Adame A, Schmidhuber S, Santic R, Schneeberger A, et al.: Active immunization against alpha-synuclein ameliorates the degenerative pathology and prevents demyelination in a model of multiple system atrophy. Mol Neurodegener 2015, 10:10.

${ }^{* *}$ Report of preclinical findings supporting the use active immunization in Parkinson's disease clincial translation. Studies have lead to multiple clincial trials testing safety, efficacy, and dosing in both multiple systems atrophy and Parkinson's disease.

59. Orgogozo JM, Gilman S, Dartigues JF, Laurent B, Puel M, Kirby LC, Jouanny P, Dubois B, Eisner L, Flitman S, et al.: Subacute meningoencephalitis in a subset of patients with $A D$ after Abeta42 immunization. Neurology 2003, 61:46-54. 
60. Reynolds AD, Kadiu I, Garg SK, Glanzer JG, Nordgren T, Ciborowski P, Banerjee R, Gendelman HE: Nitrated alpha-synuclein and microglial neuroregulatory activities. J Neuroimmune Pharmacol 2008, 3:59-74.

61. Benner EJ, Mosley RL, Destache CJ, Lewis TB, Jackson-Lewis V, Gorantla S, Nemachek C, Green SR, Przedborski S, Gendelman HE: Therapeutic immunization protects dopaminergic neurons in a mouse model of Parkinson's disease. Proc Natl Acad Sci U S A 2004, 101:9435-9440.

62. Kosloski LM, Kosmacek EA, Olson KE, Mosley RL, Gendelman HE: GM-CSF induces neuroprotective and anti-inflammatory responses in 1methyl-4-phenyl-1,2,3,6-tetrahydropyridine intoxicated mice. $J$ Neuroimmunol 2013, 265:1-10.

* Study using an inflammatory mouse model of PD to assess the ability of GM-CSF to induce a neuroprotective phenotype. Results indicate sparing of dopaminergic neurons, decreases in microglial reactivity, and the ability to modulate disease pathology within the CNS via the immune system. Findings have prompted a phase I clinical trial to test the safety of the peptide for the treatment of PD.

63. Lan Q, Fan H, Quesniaux V, Ryffel B, Liu Z, Zheng SG: Induced Foxp3(+) regulatory T cells: a potential new weapon to treat autoimmune and inflammatory diseases? J Mol Cell Biol 2012, 4:22-28.

64. Lowther DE, Hafler DA: Regulatory T cells in the central nervous system. Immunol Rev 2012, 248:156-169. 
65. Cherry JD, Olschowka JA, O'Banion MK: Are "resting" microglia more "m2"? Front Immunol 2014, 5:594.

66. Shivers KY, Nikolopoulou A, Machlovi SI, Vallabhajosula S, FigueiredoPereira ME: PACAP27 prevents Parkinson-like neuronal loss and motor deficits but not microglia activation induced by prostaglandin J2. Biochim Biophys Acta 2014, 1842:1707-1719.

67. Delgado M, Ganea D: Vasoactive intestinal peptide: a neuropeptide with pleiotropic immune functions. Amino Acids 2013, 45:25-39.

68. Fraccaroli L, Grasso E, Hauk V, Paparini D, Soczewski E, Mor G, Perez Leiros C, Ramhorst R: VIP boosts regulatory $T$ cell induction by trophoblast cells in an in vitro model of trophoblast-maternal leukocyte interaction. J Leukoc Biol 2015, 98:49-58.

69. Waschek JA: VIP and PACAP: neuropeptide modulators of CNS inflammation, injury, and repair. Br J Pharmacol 2013, 169:512-523.

70. Kelso ML, Elliott BR, Haverland NA, Mosley RL, Gendelman HE: Granulocyte-macrophage colony stimulating factor exerts protective and immunomodulatory effects in cortical trauma. J Neuroimmunol 2015, 278:162-173.

71. Schabitz WR, Kruger C, Pitzer C, Weber D, Laage R, Gassler N, Aronowski J, Mier W, Kirsch F, Dittgen T, et al.: A neuroprotective function for the hematopoietic protein granulocyte-macrophage colony stimulating factor (GM-CSF). J Cereb Blood Flow Metab 2008, 28:29-43. 
72. Lacan G, Dang H, Middleton B, Horwitz MA, Tian J, Melega WP, Kaufman

DL: Bacillus Calmette-Guerin vaccine-mediated neuroprotection is associated with regulatory T-cell induction in the 1-methyl-4-phenyl1,2,3,6-tetrahydropyridine mouse model of Parkinson's disease. $J$ Neurosci Res 2013, 91:1292-1302.

73. Schuhmann MK, Kraft P, Stoll G, Lorenz K, Meuth SG, Wiendl H, Nieswandt B, Sparwasser T, Beyersdorf N, Kerkau T, et al.: CD28 superagonistmediated boost of regulatory $\mathrm{T}$ cells increases thrombo-inflammation and ischemic neurodegeneration during the acute phase of experimental stroke. J Cereb Blood Flow Metab 2015, 35:6-10.

74. Na SY, Mracsko E, Liesz A, Hunig T, Veltkamp R: Amplification of regulatory $\mathrm{T}$ cells using a CD28 superagonist reduces brain damage after ischemic stroke in mice. Stroke 2015, 46:212-220.

75. Chan A, Yan J, Csurhes P, Greer J, McCombe P: Circulating brain derived neurotrophic factor (BDNF) and frequency of BDNF positive T cells in peripheral blood in human ischemic stroke: Effect on outcome. $J$ Neuroimmunol 2015, 286:42-47.

76. Zhao W, Beers DR, Liao B, Henkel JS, Appel SH: Regulatory T lymphocytes from ALS mice suppress microglia and effector $T$ lymphocytes through different cytokine-mediated mechanisms. Neurobiol Dis 2012, 48:418-428.

77. Beers DR, Henkel JS, Zhao W, Wang J, Huang A, Wen S, Liao B, Appel SH: Endogenous regulatory $\mathrm{T}$ lymphocytes ameliorate amyotrophic 
lateral sclerosis in mice and correlate with disease progression in patients with amyotrophic lateral sclerosis. Brain 2011, 134:12931314.

78. Henkel JS, Beers DR, Wen S, Rivera AL, Toennis KM, Appel JE, Zhao W, Moore DH, Powell SZ, Appel SH: Regulatory T-lymphocytes mediate amyotrophic lateral sclerosis progression and survival. EMBO Mol Med 2013, 5:64-79.

** This study, along with many past studies, reports findings that demonstrate the neuroprotective effects elicited by regulatory $T$ cells in a neurodegenerative disorder apart from Parkinson's disease.

79. Chung ES, Lee G, Lee C, Ye M, Chung HS, Kim H, Bae SS, Hwang DS, Bae H: Bee Venom Phospholipase A2, a Novel Foxp3+ Regulatory T Cell Inducer, Protects Dopaminergic Neurons by Modulating Neuroinflammatory Responses in a Mouse Model of Parkinson's Disease. J Immunol 2015. 


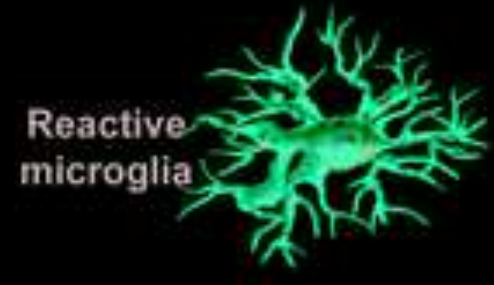

0
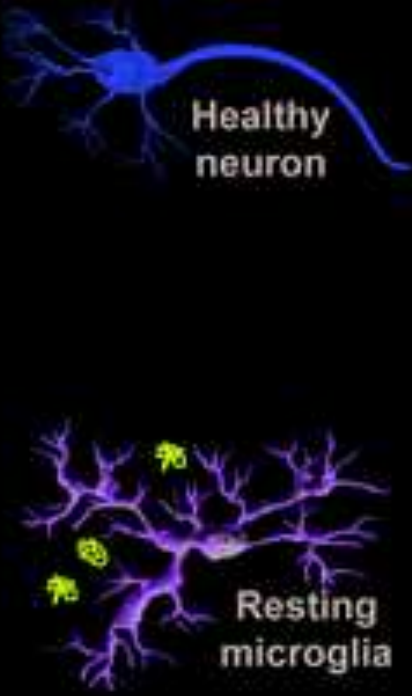

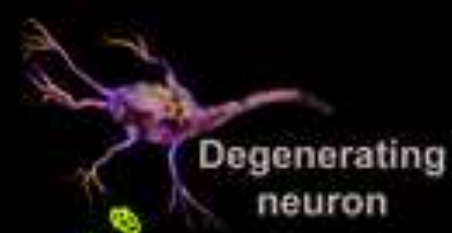

thes

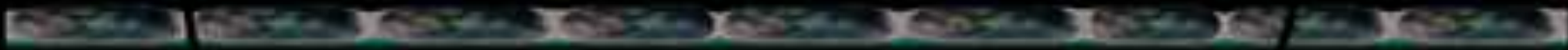

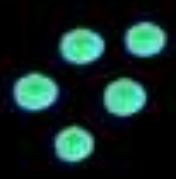

40 急

\section{Healthy \\ neuron}
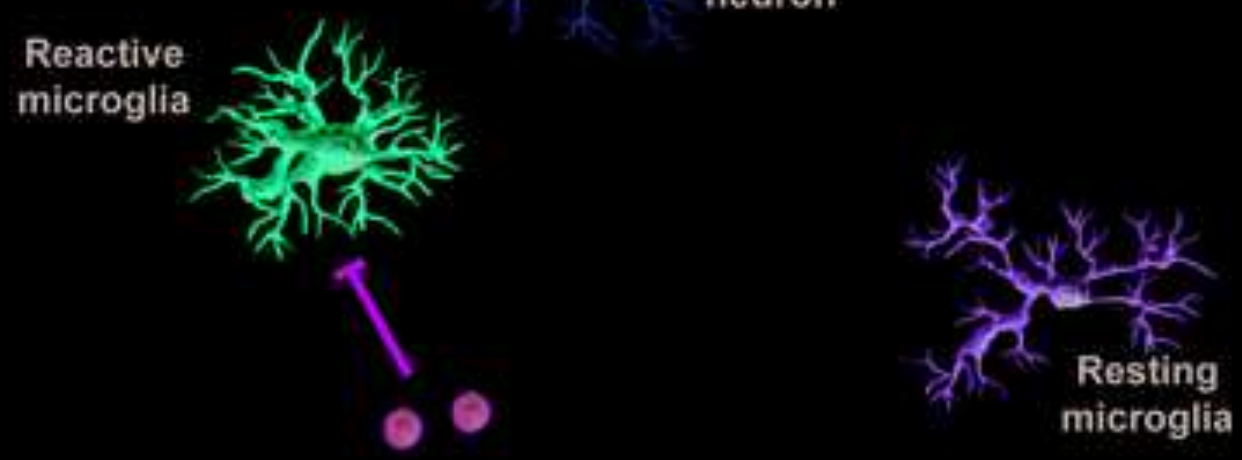

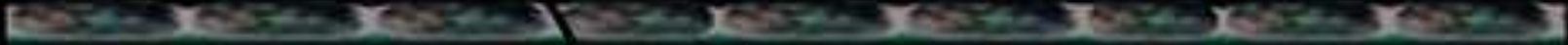
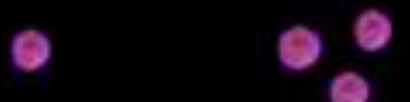